T. Hagfors: Doesn't the theory presented here grossly overestimate the effects of shadowing, since the shadowed regions would not be inclined favorably for reflection anyway?

Answer: No; cf. sec III and IV of Beckmann [1965b].
R. M. Goldstein: It seems as though the returned power does not go to zero at the limb, in your expression.

Answer: It goes to zero, cf. (14) for $\theta \rightarrow \pi / 2$.

(Paper 69D12-621)

\title{
A Note on the Radio Reflectivity of the Lunar Surface
}

\author{
A. Giraud \\ National Center of Telecommunicational Studies, CDS Department, Issy les Moulineaux (Seine) \\ To the extent that scattering phenomena can put boundary conditions on properties of the surface, \\ the results obtained by the use of radar have provided less information on the lunar surface than the \\ passive radio observations. Where the latter have given information such as on the refractive index \\ or the thermal resistivity, the interpretation of the lunar radar echoes has dealt principally with the \\ character of the geometry of the reflected surface. Once this aspect is established, it is possible to \\ obtain the reflection coefficient at normal incidence, given by Fresnel's formula,

$$
\frac{1-\sqrt{K^{\prime}+i K^{\prime \prime}}}{1+\sqrt{K^{\prime}+i K^{\prime \prime}}}
$$ \\ where $K^{\prime}$ and $K^{\prime \prime}$ are the real and imaginary parts of the complex relative permittivity. \\ 'In this way one finds $K^{\prime} \simeq 2.7$ at decimeter wavelengths. The same coefficient of reflectivity \\ obtained at centimeter wavelengths by Russian radio astronomers, through the surface emissivity \\ and Kirchhoff's Law, give us $K^{\prime} \simeq 1.5$. This difference between the properties of the lunar surface \\ was attributed to the greater penetration of the longer waves. \\ In this study we propose to explain such a variation of the coefficient of reflectivity with wavelength, \\ by using only radar data. We will quantitatively explain this variation by the use of a model where \\ the lunar surface does not consist of abrupt discontinuities - where the reflective properties may be \\ explained by (1) at wavelengths greater than a few meters.
}

\section{The Spectra of the Lunar Reflectivity at Short Wavelengths}

\subsection{General}

The published results of lunar radar observations range from wavelengths of about $1 \mathrm{~cm}$ to more than $10 \mathrm{~m}$. They show a progressive change in the mechanism of reflection, being diffused at millimeter wavelengths and semitransparent at decameter wavelengths (fig. 1). At the same time, the fraction of the returned energy, or the effective scattering cross section $\sigma$, seems to increase by a factor of the order of two (fig. 2) between $10 \mathrm{~cm}$ and $10 \mathrm{~m}$. We can write

$$
\sigma=\pi a^{2} r g
$$

Where $a$ is the radius, $g$ represents the "gain" due to the geometric characteristics of the surface, and $r$ is the mean "albedo" corresponding to the intrinsic electromagnetic properties of the material responsible for it.

For the extreme cases of a perfectly smooth sphere and of one completely diffused (according to Lambert's law), the factors $r$ and $g$ are independent. Let us assume that the constituents have the same dielectric constant $K$ to a depth much larger than the wavelengths in use.

In the first case we have

$$
\sigma_{\text {smooth }}=\pi a^{2}|R|^{2},
$$

where $R$ is Fresnel's coefficient for normal incident given by (1). For $K=3$, for example, we have

$$
\sigma_{\text {smooth }}=0.072 \pi a^{2} .
$$

In the second case,

$$
\sigma_{\text {Lambert }}=\frac{8}{3} \pi a^{2}|\bar{R}|^{2},
$$

where $|\bar{R}|$ represents a mean reflection coefficient averaged for all incident angles and all polarizations. It is possible to show numerically that $|\bar{R}|^{2}=0.125$; therefore

$$
\sigma_{\text {Lambert }}=0.33 \pi a^{2} .
$$

As the wavelength becomes longer, the effective lunar cross section, instead of increasing, decreases by a factor of 4 or 5 at the same time that the specular 
character of the reflection increases. Qualitatively we can conclude that the intrinsic reflectivity of the lunar surface decreases with $\backslash$ frequency.

The rigorous expression for $g$ of (2) in the case of a diffuse reflection is very complex; we will examine only the optically geometrical approximation.

\subsection{The Quasi-Specular Component}

The angular spectrum of a reflected field by an irregular surface decreases at first rapidly with the angle of incidence $\theta$, then generally, a diffuse component appears proportional to $\cos ^{3 / 2}=\theta$ (Lambert's law) or to $\cos \theta$ (Lommel-Seeliger) (fig. 1). Application of the Huygens-Kirchhoff principle to slightly undulating surface models permits to a certain extent taking into account the first component, called the quasi-specular. Contrary to what goes on in the presence of irregularities of the same order as the wavelength, it is only the surface elements which are conveniently oriented that contribute efficiently to the backscattering. It can be shown that the effective cross section of a sphere whose surface can undergo such an analysis, such as a smooth sphere, is given by (3), and the depolarization of the reflected wave is negligible [Beckmann and Spizzichino, 1963; Hagfors, 1964 .

The problem then consists of determining what fraction of the effective total cross section (quasi-

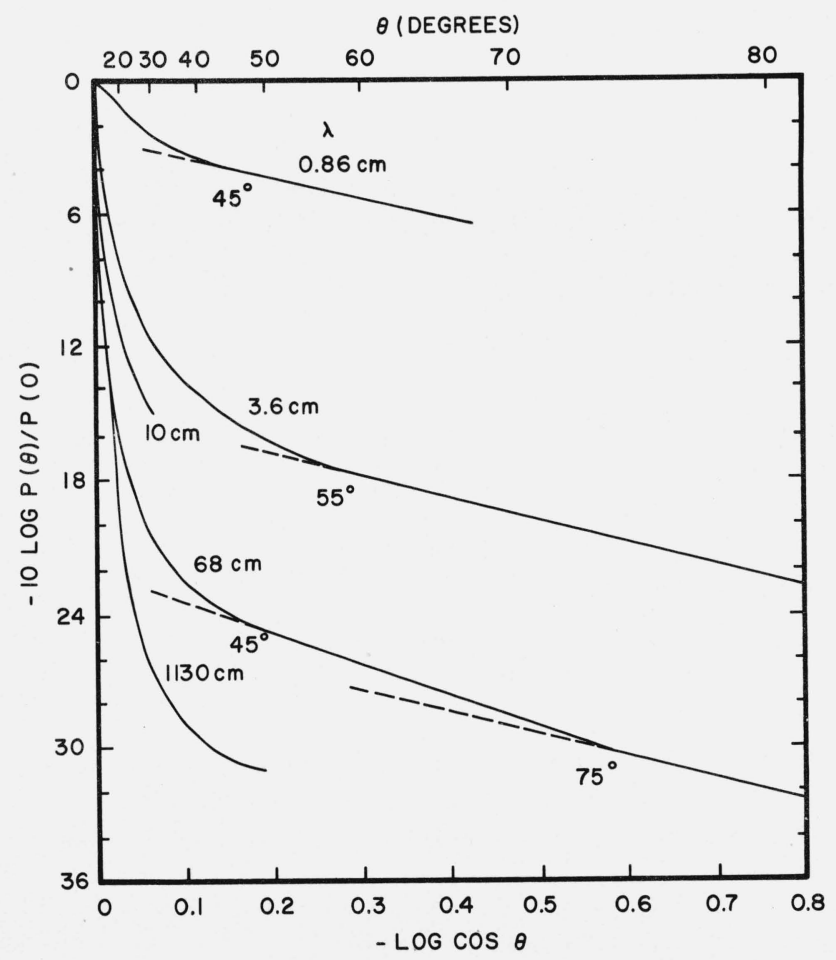

FIGURE 1. Normalized reflected power by the moon as a function of angle of incidence [Evans and Pettengill, 1963a, b; Lynn et al., 1964; Davis and Rohlfs, 1964].

The components proportional to $\cos \theta$ and $\cos ^{3 / 2} \theta$ are indicated.

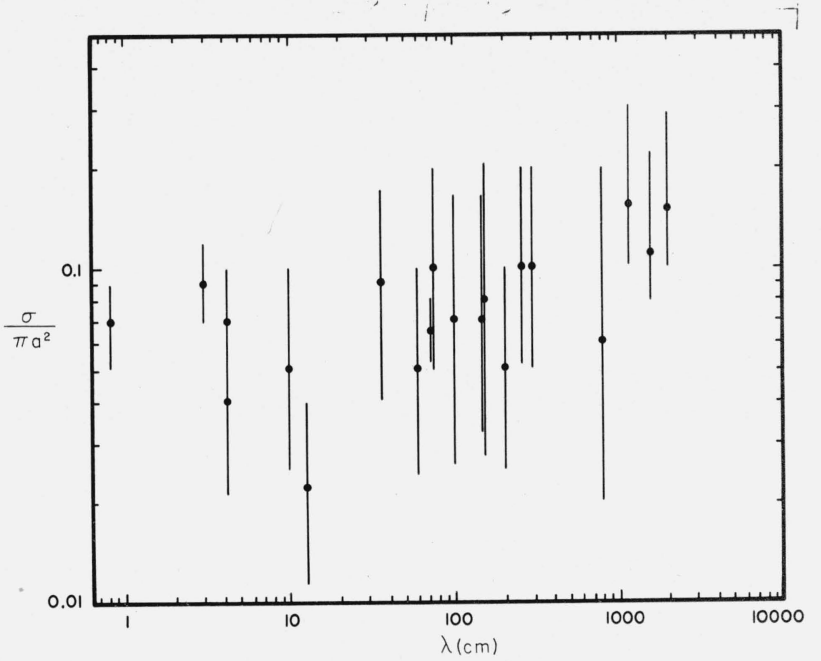

FiguRE 2. Total effective radar cross section of the moon as a function of wavelength [Evans and Pettengill, 1963a, b; Lynn et al., 1964; Davis and Rohlfs, 1964].

specular + diffuse) has to be attributed to each component. In the absence of a quasi-specular model which is in sufficient agreement with experimental results, we can try in some way to subtract the diffuse component. To estimate this, the most accurate method is that used by Rea et al., [1964] which uses the depolarized portion of the returned energy. Unfortunately polarization measurements were made only at a wavelength of $68 \mathrm{~cm}$. The results obtained by Rea et al., do not differ substantially from those obtained by subtracting the components that obey Lambert's and Lommel-Seelinger's laws [Evans and Pettengill, 1963]. We therefore adopt this procedure, thus introducing an uncertainty of 20 percent. We then obtain for the ratio $\sigma_{q} / \sigma$ the fraction of the energy returned by a quasi-specular mechanism

\begin{tabular}{cccccc}
$\lambda(\mathrm{cm}): 0.86: 3.6: 10: 68: 1130$ \\
\hline$\sigma_{q} / \sigma(\%): 15 \pm 10: 50 \pm 10: 60 \pm 10: 80 \pm 10: 90 \pm 10$
\end{tabular}

At $8.6 \mathrm{~mm}$ more than half of the lunar surface contributes to the diffused reflection, so it is doubtful that the quasi-specular model can be used at this wavelength.

The values of $\sigma$ that we will use are those given in figure 2 .

\subsection{The Reflective Coefficient}

The results of the application of (3) to the fraction of the effective cross section attributed to the quasispecular component at the four wavelengths under consideration are given in figure 3 . The error bars take into account the uncertainty of the measurements of the total effective cross section and the uncertainty in our determination of the retained fraction. 


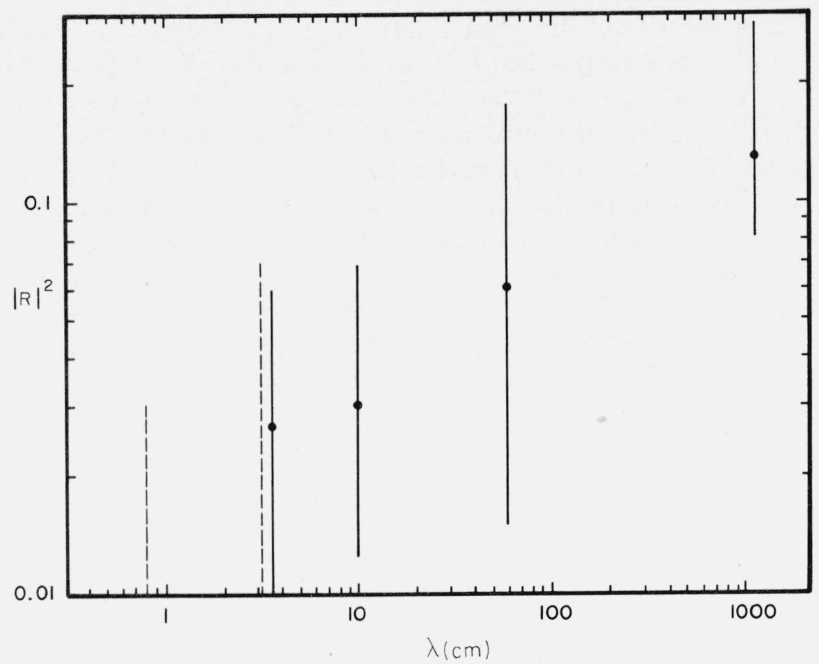

FigURE 3. The reflection coefficient of the lunar surfaces as a function of wavelength deduced from the quasi-specular component of echoes received at 3.6, 10,68, and $1130 \mathrm{~cm}$.

The reflective coefficient deduced from the emissivity at 0.8 centimeters and $3.2 \mathrm{~cm}$ [Solomonovich and Losovskii, 1963; Soboleva, 1963; Krotikov and Troitsky, 1963]. is also indicated.

The values of the reflection coefficient at 8-mm and $3.2-\mathrm{cm}$ wavelengths are those obtained by Salomonovich and Losovskü [1963] and Krotkov and Troitsky [1963] from calculations of the radio emissivity of the lunar surface. Similarly, at $3.2 \mathrm{~cm}$ the value indicated by a cross corresponds to that obtained by Soboleva from a study of the polarization of the thermal emissivity. It can be seen that there is good agreement.

Although a constant reflection coefficient of about 7 percent, which corresponds from (1) to a dielectric constant of 3 if $K^{\prime \prime}$ is negligible, cannot be absolutely excluded; it seems that a significant increase exists between a wavelength of a few centimeters and several meters. It would be interesting to obtain the effective lunar cross section at longer wavelengths to confirm this result, but such measurements cannot be performed except from above the Earth's ionosphere.

\section{Interpretation: The Superficial Layer}

\subsection{Choice of an Interpretation}

If we assume a homogeneous lunar surface relative to the wavelength considered, it refers to the discussion of section 1.2 that the coefficient of reflectivity $R$ is given by Fresnel's formula (1). In this case all the variation with frequency is attributed to the interaction of the applied alternating field and the microscopic constitution of the surface. This interaction is expressed by the values of the dielectric constant $K^{\prime}$ and the loss factor $K^{\prime} / K^{\prime \prime}$.

In the frequency range studies, these parameters rarely change. If the factor of the losses is effectively constant, the electric conductivity is proportional to the frequency. This law has in fact been verified at centimeter wavelengths by the decrease of the variable component of the lunar radio temperature [Giraud, 1962; Troitsky, 1963]. On the other hand, it is certain that the polarization phenomena which is responsible for losses, and the dielectric constant are at least as sensitive to temperature as the frequency, which makes illusory the interpretation based on molecular-scale considerations.

On the contrary, a microscopic explanation presents itself, mainly because we want to form a model valid in the entire observable electromagnetic spectrum. Hapke and Van Horn [1963] have shown that the optical properties of the lunar surface correspond to materials that have a very loose structure which are susceptible to sinking under their own weight. This vesicular structure is also deduced from temperature observations in the infrared. Finally the thermal observations at millimeter and centimeter wavelengths can be interpreted better if we assume that the density increases with depth by the use of a homogeneous model. These independent conclusions make us propose reflective laws in stratified media, in place of (1).

\subsection{Reflective Coefficient of a Density Gradient Layer (Comparison)}

It has been shown by Born and Wolf [1959] that a stratified medium, that is to say, one whose properties (here the permittivity $K$, assumed real, and the permeability $\mu$ ) are constant in planes perpendicular to a certain fixed axis, is completely determined from the point of view of a plane electromagnetic wave by a "characteristic matrix" of the order of 2 . In the case of a normal incidence to the stratified plane, a first approximation of this matrix is

$$
\left[\begin{array}{cc}
1 & \frac{-2 \pi i}{\lambda} \int_{0}^{d} \mu(x) d x \\
\frac{-2 \pi i}{\lambda} \int_{0}^{d} K(x) d x & 1
\end{array}\right]
$$

where the limits of the integration extend to all the depths of the stratified layer.

The reflection coefficient of such a layer, placed between two homogeneous and semi-infinite media which have infinite permittivity and permeability $k_{0}$, $\mu_{0}$ and $k_{1}, \mu_{1}$ respectively, is given by $|R|^{2}$ as

$$
R=\frac{\left(m_{11}+m_{12} p_{1}\right) p_{0}-\left(m_{21}+m_{22} p_{1}\right)}{\left(m_{11}+m_{12} p_{1}\right) p_{0}+\left(m_{21}+m_{22} p_{1}\right)}
$$

where $p_{0}=\sqrt{\frac{K_{0}}{\mu_{0}}}$ and $p_{1}=\sqrt{\frac{K_{1}}{\mu_{1}}}$, and $m_{i j}$ are the characteristic matrix elements. 
If the first medium is space, we get

$$
R=\frac{\left(1-\sqrt{K_{1}}\right)-i \frac{2 \pi d}{\lambda}\left(\sqrt{K_{1}}-\bar{K}\right)}{\left(1+\sqrt{K_{1}}\right)-i \frac{2 \pi d}{\lambda}\left(\sqrt{K_{1}}+\bar{K}\right)}
$$

where $\bar{K}=\frac{1}{d} \int_{0}^{d} K(x) d x$ represents the mean dielectric constant of the layer.

It can be seen that for $\lambda \gg d$ the reflection takes place as though the layer did not exist. For $\lambda \ll d$ the layer reflects as if it were composed of a homogeneous material of dielectric constant $\bar{K}^{2} / K_{1}$.

If we assume that these limiting cases are attained for the Moon at decameter and millimeter wavelengths respectively, the results, given in figure 3 , indicate that $K_{1} \simeq 6$ and $K \simeq 3.5$.

To estimate the depth of the layer it is necessary to postulate the variation in $K(x)$ with depth. We have examined the linear case, where $K(0)=1, K(d)=K_{1}$. This result shows that the layer represents the porous state of the material that it covers, and that the porous state decreases uniformly with depth. The curves in figure 5 and 6 show that the variation of $R$ of figure 3 corresponds to a depth $d$ between 10 and $50 \mathrm{~cm}$.

\subsection{Discussion}

The results that have been obtained apply to the surface elements of the Moon where the normal is directed towards the Earth (see sec. 1.2). The variation of the angle $\theta$ between the normal and the vertical brings two comments. First, the hypothesis of a density gradient perpendicular to the wave plane is only justified for small values of $\theta$, that is, the case for a quasi-specular echo. Therefore, the angular spectrum of the reflected field is not determined in

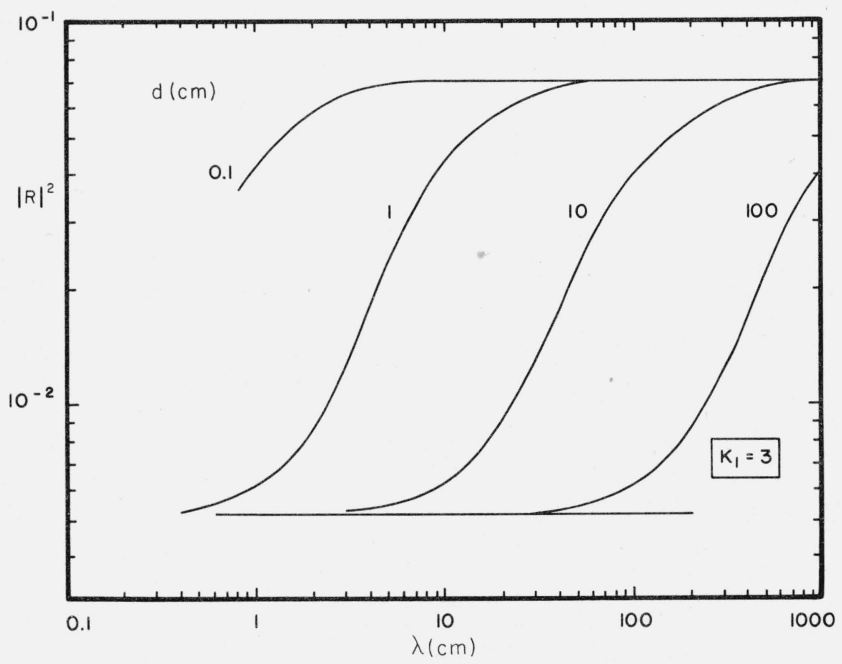

FIGURE 4. The reflection coefficient of a stratified layer as a function of wavelength; $\mathrm{K}_{1}=$ dielectric constant of a compact material; $\mathrm{d}=$ thickness of layer for a linear variation of the dielectric constant with depth. this case by the distribution of the slopes. Second, our conclusions apply only to accidental regions, that is to say, to regions where slopes exist; here regions are greater than several meters, and maybe this applies to the entire lunar surface.

The choice of the linear increase of the dielectric constant with depth is naturally artificial, but serves to stimulate ideas. It is not difficult to foresee the results produced by other models; for example, the curves in figures 4,5 , and 6 could correspond to a homogeneous superficial layer where the value of the dielectric constant is half that of the sublayer. If the gradient of $K$ increases with depth, the thickness of the layer must be greater in order to explain the variation of the reflectivity. Hapke and Van Horn [1963] mention a thickness of about $60 \mathrm{~cm}$ for the Moon for the settling of fine particles that they studied in the laboratory.

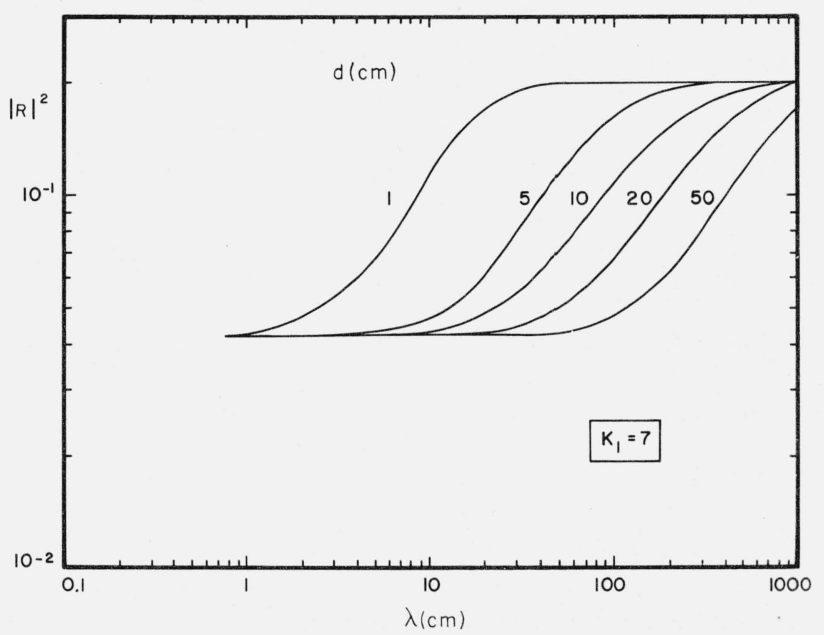

FIGURE 5. The reflection coefficient of a stratified layer as a function of wavelength; $\mathrm{K}_{1}=$ dielectric constant of a compact material; $\mathrm{d}=$ thickness of layer for a linear variation of the dielectric constant with depth.

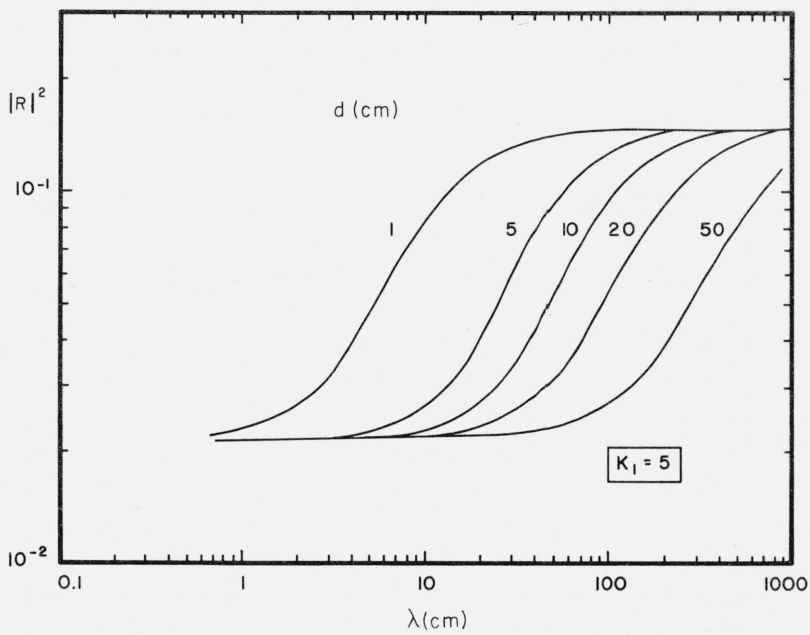

Figure 6. The reflection coefficient of a stratified layer as a function of wavelength; $\mathrm{K}_{1}=$ dielectric constant of a compact material; $\mathrm{d}=$ thickness of layer for a linear variation of the dielectric constant with depth. 
It is worth mentioning the abnormal reflection associated with the crater Tycho [Pettengill and Henry, 1962], where the reflection was about five times greater than its surroundings at $440 \mathrm{Mc} / \mathrm{s}$. Figure 6 indicates that this corresponds to a thickness of the lower layer of $1 \mathrm{~cm}$, if our interpretation is correct.

We can remark that, dealing with the nature of compact materials, a dielectric constant of 6 and 7 is in good agreement with that of tektites [Olte and Siegel, 1960].

\section{Conclusions}

We have shown that the observed variation of the effective scattering cross section of the Moon suggest an increase of the reflectivity with wavelength, although we cannot disregard the possibility of a constant reflective coefficient of a homogeneous surface structure.

If this variation is due to a superficial layer, where the density increases with depth, the following conclusions can be stated:

The thickness of the layer is of the order of a few tens of centimeters, at least for a good part of the slopes that are responsible for the quasi-specular component of the received echos.

The dielectric constant of the material that the layer is formed of is higher than 5 at the compact state.

\section{References}

Beckmann, P., and A. Spizzichino (1963), The Reflection of Electromagnetic Waves from Rough Surfaces (Pergamon Press, Oxford, Macmillan Co., New York, N.Y.).

Born, M., and E. Wolf (1959), Principles of Optics (Pergamon Press, Oxford).
Davis, D. G., and D. C. Rohlfs (1964), Lunar radio reflection properties at decameter wavelengths, J. Geophys. Res. 69, No. 15, $3257-3262$.

Evans, J. V., and G. H. Pettengill (1963a), The scattering behavior of the Moon at wavelengths of 3.6, 68 and $784 \mathrm{~cm}$, J. Geophys. Res. 68, No. 2, 423-447.

Evans, J. V., and G. H. Pettengill (1963b), The radar cross-section of the Moon, J. Geophys. Res. 68, No. 17, 5098-5099.

Giraud, A. (1962), Characteristics of the Moon's surface layer. An analysis of its radio emission, Astrophys. J. 135, No. 1, 175.

Hagfors, T. (1964), Backscattering from an undulating surface with applications to radar return from the Moon, J. Geophys. Res. 69, No. 18, 3779-3784.

Hapke, B., and H. Van Horn (1963), Photometric studies of comples surface, J. Geophys. Res. 68, No. 15, 4545-4570.

Krotikov, V. D., and V. S. Troitsky (1963), The emissivity of the Moon at centimeter wavelengths, Soviet Astronomy, AJ 6, No. 6 , 1089-1093.

Lynn, V. L., M. D. Sohigian, and E. A. Crocker (1964), Radar observations of the Moon at a wavelength of $8.6 \mathrm{~mm}$, J. Geophys. Res. 69, No. 4, 781-783.

Olte, A., and K. M. Siegel (1960), Distinction between the electromagnetic constants of Tektites and Lybian Desert glass and their effect on lunar surface theory, Astrophys. J. 133, No. 2, 706-717.

Pettengill, G. H., and J. C. Henry (1962), Enhancement of radar reflectivity associated with the lunar crater Tycho, J. Geophys. Res. 67, No. 12, 4881-4885.

Rea, D. G., N. Hetherington, and R. Mifflin (1964), The analysis of radar echoes from the moon, J. Geophys. Res. 69, No. 24, 52175223.

Soboleva, N. S. (1963), Measurement of the polarization of lunar radio emission on a wavelength of $3.2 \mathrm{~cm}$, Soviet Astronomy. AJ 6, No. 6, 1124-1126.

Solomonvich, A. E.. and B. Ya. Losovskii (1963), Radio brightness distribution on the lunar disk at $0.8 \mathrm{~cm}$. Soviet Astronomy, AJ 6, No. 6, 1074-1082.

Troitsky, V. S. (1963), Nature and physical state of the surface layer of the moon. Soviet Astronomy. AJ 6, No. 1.

\title{
Moon Distance Measurement by Laser
}

\author{
A. Orszag \\ École Polytechnique, Paris
}

\section{Introduction}

It seems hardly necessary to recall the main characteristics of the ruby laser, the first to have functioned and still the most widely used today. These characteristics are essentially:

(a) A very monochromatic light,

(b) a very narrow beam,

(c) lastly, and in particular with the so-called $Q$ switched lasers, an emission effected by very short pulses of which the peak power attains considerable values.

The small aperture of the radiated beam, in conjunction with the very high peak power of the emission,

\footnotetext{
1 This work is supported by the following organizations: C.N.E.S., C.N.E.T., D.G.R.S.T D.R.M.E., and the committee for Laser Atmospheric and Spatial Applications, under the direction of Pr. P. Grivet. The project is to be conducted at the Ecole Polytechnique and the Pic du Midi Observatory, under the supervision of Pr. Rosch.
}

means that the laser represents a source of considerable brightness, reaching, for example, $10^{10} \mathrm{~W} / \mathrm{cm}^{2} /$ sterad.

A light source presenting such properties could certainly not fail to attract the attention of all those particularly interested in the location and ranging of distant objects.

The first realizations in this field were infantry and tank telemeters, destined to measure distances not exceeding a few kilometers. Then, as laser power increased, the measurement of far greater distances came to be attempted, for example, that of a satellite, taken successfully a short time ago in the United States [Plotkin, 1963] and in France [Bivas, 1965]. It was therefore tempting to try to measure, using a laser as light source, the Earth-Moon distance, already measured by radar [Yaplee et al., 1963]. 